DOI: $10.2478 / \mathrm{v} 10282-012-0008-\mathrm{z}$

\title{
Brain abscesses: management and outcome analysis in a series of 84 patients during 12 year period
}

\author{
M. Gorgan, Angela Neacsu, Narcisa Bucur, V. Pruna, Catalina Lipan, \\ Aurelia Mihaela Sandu, Catioara Fanica Cristescu
}

First Neurosurgical Clinic, Fourth Neurosurgical Department, Clinic Emergency

Hospital "Bagdasar-Arseni", Bucharest

\begin{abstract}
Authors analyze 84 cases of brain abscesses operated between January 2000 and December 2011, in the Fourth Neurosurgical Department by the same surgical team. We underline the general series profile: the mean age was 42.96 years (range: $11-75$ years old), $72.62 \%$ were males, association with heart defects in seven cases $(8.33 \%)$, positive bacteriological examination for germs in only 37 cases (44.04\%), all negative for HIV infection. The median number of days to diagnosis was 9. Most frequent clinical presentations included headache $(40.47 \%)$, fever $(35.71 \%)$, focal neurologic deficits $(29.76 \%)$, increased intracranial pressure $(28.57 \%)$ and seizures (11.90\%).

The majority of cases $(76.19 \%, n=64)$ presented a supposed medical condition favoring dissemination of a previous infection: malnutrition, tuberculosis, chronic alcoholism, chronic liver malady, neglected dental or ear infections, and only 5 cases $(5.95 \%)$ had been diagnosed with secondary immunodeficiency syndromes following autoimmune systemic diseases.

According to our treatment policy all cases except for two (treated by puncture and aspiration) benefited of open surgery and total removal of the lesions, without local recurrence. Outcome was favorable in $82.14 \% \quad(n=69) \quad$ of subjects. General
\end{abstract}

morbidity was $26.19 \%$, and mortality stood at $7.14 \%$. Six cases remained with permanent motor deficit (7.14\%) and four $(4.76 \%)$ with controllable seizures. Out of a total of $33.33 \%(n=28)$ of complications, $64.28 \%$ were due to medical causes.

Follow-up had been extended up to three years for at least $2 / 3$ of patients, who resolved in time medical or surgical conditions which determined cerebral dissemination of the infection. Despite of a poor medical and biological condition, the patients with brain abscess outside of HIV infections benefit from neurosurgical adequate treatment, and if supportive medical and general therapy is continued and sustained, the healing and survival in good neurological status is the rule. Hematogenous spread and advance age were predictors of poor prognosis. Our findings are similar to the results of recent works, although in our series, there is a higher frequency of aerobe germs.

Key words: brain abscess, surgical treatment, abscess complications.

\section{Introduction}

Brain abscess and subdural empyema are serious infections which can be metastasis of chronic suppurative diseases (bronchiectasis, lung or abdominal abscesses) or a congenital cardiomyopathy, from an open head injury or from 
neurosurgical procedures, but they are more frequently seen in healthy adults suffering from chronic sinusitis or otitis (2).

Brain abscesses have proven to be one of the disease entities that the neurosurgeon can treat most successfully. This has not always been the case; however, as until only recently brain abscesses have had extremely high morbidity and mortality rates. Technological advances including the development of antibiotics, improved bacteriological identification techniques, and, most important, development of computed tomography (CT) have changed the prognosis dramatically $(2,9,10,12,16)$.

The uncomplicated brain abscesses early recognized, accurately localized and promptly operated upon is one of the most satisfactory of the intracranial lesions. One might almost conclude that in uncomplicated abscesses of the brain operated at a fair period, recovery ought to be the rule.

Despite the prompt use of modern antibiotics in the treatment of predisposing causes of cerebral abscesses, the incidence of this disease has changed little and, if anything, seems to be increasing. This may be a result of the increase in the immunosuppressed population (7). These patients, however, usually do not present with abscesses cause by pyogenic bacteria but instead with those caused by fungi, parasites, or other opportunistic organisms. This increase is more likely due to the increased ability to make the diagnosis with modern imaging techniques.

This report is based on our experience with brain abscesses surgically managed in 84 patients over a 12-year period and analyzes the factors with an impact on morbidity, mortality, and outcome.

\section{Material and methods}

Authors analyze 84 consecutive cases with brain abscesses, operated between January 2000 and December 2011, in the Fourth Neurosurgical Department of the Clinical Emergency Hospital of Bucharest, by the same surgical team, the soonest possible after admission, non-randomized.

Demographic data, clinical presentation, neurological status at admission, number of days to diagnosis, anatomical location and number of lesions, predisposing factors, etiology, treatment, complications and outcome were assessed. A predisposing factor was defined as any event or condition that was directly related to the onset of brain abscess. Routes of transmission were classified as infection originating through hematogenous spread, contiguous spread, open head injury, neurosurgical procedures, or unknown causes. Outcome was appraised through the Glasgow Outcome Scale. Complications were defined as any deviation from normal postoperative course occurring within 30 days following surgery, and they were classified as surgical or medical (adverse events that are not directly related to surgery).

Mainly the diagnosis was completed after contrast CT examinations, except for 10 patients with MRI. The medium size of abscess was appreciated using CT scan: 58 cases $(69.04 \%)$ between $3-4.5 \mathrm{~cm} ; 19$ cases (22.61\%) between $4.6-6 \mathrm{~cm}$, and 7 cases $(8.33 \%)$ over $6 \mathrm{~cm}$ in diameter. Ring enhancement was firmly represented in all cases except one case with multiple abscesses, suggesting a long clinical course and indicating a good result after open surgery. Preoperative investigations included: chest x-rays, blood tests, urine samples, EKG, and bacteriological examinations when possible. 
DOI: $10.2478 / \mathrm{v} 10282-012-0008-\mathrm{z}$

According to our treatment policy all cases except for two (treated by burr-hole and aspiration) benefited of open surgery and lesion removal through craniotomy, with resection of the capsule, without local recurrence. We used decompressive craniotomy in four patients. Patients treated by repetitive puncture and aspiration were in poor medical condition (one had endocarditis and Fallot tetrad and one suffered from ARDS and renal failure), with lesions located in eloquent motor area.

\section{Results}

In our series, the average age was 42.96 years (range: $11-75), 72.62 \%(n=61)$ were males and $27.38 \%(n=23)$ females. The median number of days to diagnosis was nine (range: 2-45 days). The majority of cases $(76.19 \%$ - 64 cases) presented a supposed medical condition favoring dissemination of a previous infection: neglected dental $7.14 \%(n=6)$, paranasal sinuses $4.76 \%(n=4)$, middle ear or mastoid infections $21.42 \% \quad(n=18)$, lung abscess $2.38 \%(n=2)$, malnutrition $11.9 \%(n=10)$, tuberculosis $8.33 \%(n=7)$, chronic ethanol abuse $9.52 \%(\mathrm{n}=8)$, chronic liver disease $4.76 \%(\mathrm{n}=4)$, and only 5 cases $(5.95 \%)$ weren diagnosed with secondary immunodeficiency syndromes following autoimmune systemic diseases (lupus, chronic leukemia, diabetes), all negative for HIV infection or intravenous abuse. Association with heart defects was present in seven cases $(8.33 \%)$.

78 cases $(92.85 \%)$ presented a single intracerebral abscess and six cases had multiple lesions (7.14\%). 77 abscess (91.66\%) were located supratentorial and seven were infratentorial $(8.33 \%)$.

Patients presented on admission: headache $(40.47 \%, \mathrm{n}=34)$, fever $(35.71 \%$, $\mathrm{n}=30$ ), focal neurologic deficits $(29.76 \%$, $\mathrm{n}=25)$, increased intracranial pressure $(28.57 \%, \mathrm{n}=24)$ and seizures $(11.90 \%$, $\mathrm{n}=10)$.
The most common mechanisms of infection included hematogenous spread (35.71\%, $\mathrm{n}=30)$, contiguous spread $(33.33 \%, \quad \mathrm{n}=28), \quad$ post-neurosurgical procedures $(13.09 \%, \mathrm{n}=11)$, and open head injury $(5.95 \%, n=5)$. The route of transmission remained unknown in $23.8 \%$ of patients $(n=20)$.

Many patients received antibiotics before surgery, being treated in other services. This is one of the reasons that positive bacteriological examination for germs in our series was found in only 37 cases (44.04\%). Preoperative antibiotherapy correlated significantly with the occurrence of sterile cultures. Out of the total of 37 patients with cultures positive for germs, most common responsible bacteria were staphylococcus in 17 cases (20.23\%), proteus in 8 cases $(9.52 \%)$, and klebsiella in 5 cases $(5.95 \%)$.

Outcome was favorable in $82.14 \%$ $(n=69)$ of the subjects. Six cases remained with permanent motor deficit (7.14\%), and only one patient had new neurological deficits following surgery. Four patients $(4.76 \%)$ remained with controllable seizures. General morbidity was $26.19 \%$ $(n=22)$, and mortality stood at $7.14 \%$ $(n=6)$. Out of a total of $33.33 \% \quad(n=28)$ complications, $64.28 \%$ were secondary to medical causes $(66.66 \%, \mathrm{n}=4$, of deaths were due to medical causes and were not directly related to surgery).

Age was related to favorable postoperative outcome. The mean age of patients with good outcome was $40.57 \pm 16.25$ years, and $55.48 \pm 20.34$ years in the case of subjects with poor GOS scores. Compared with other mechanisms of infections, hematogenous spread correlated with unfavorable outcome. We recorded no recurrences, and follow-up had been extended up to three years for at least $2 / 3$ of patients, who resolved in time medical or surgical conditions which determined cerebral dissemination of the infection. 


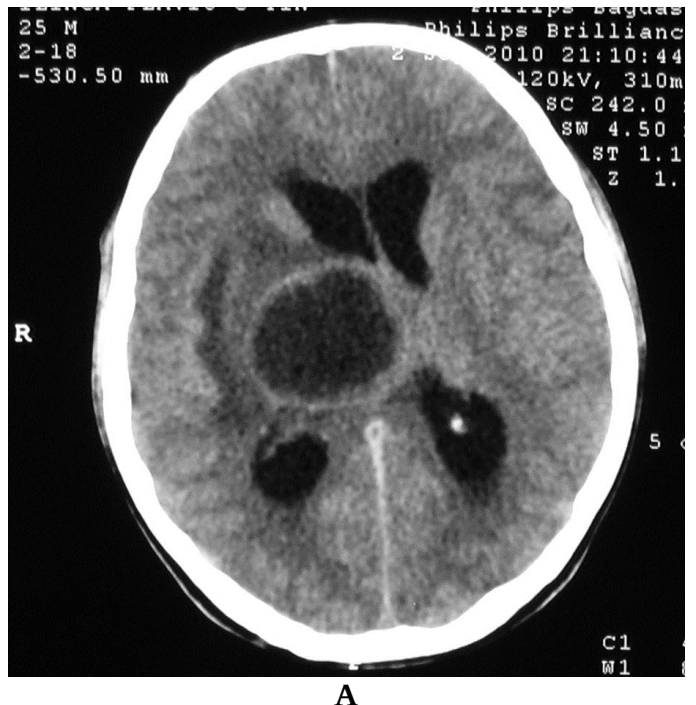

A

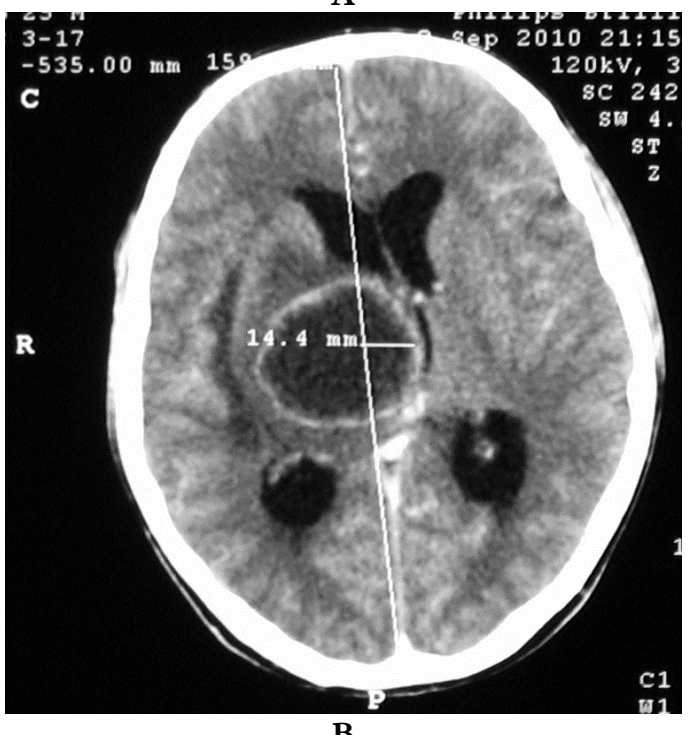

B

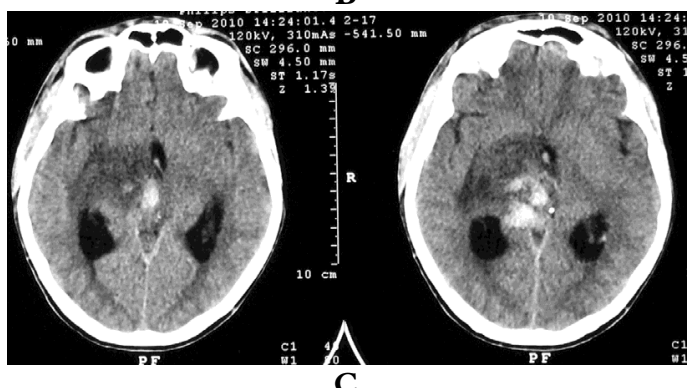

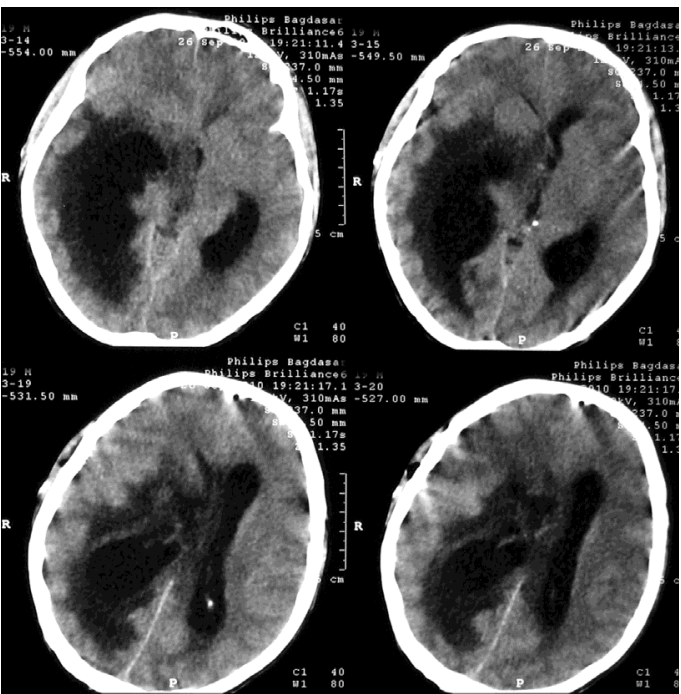

D

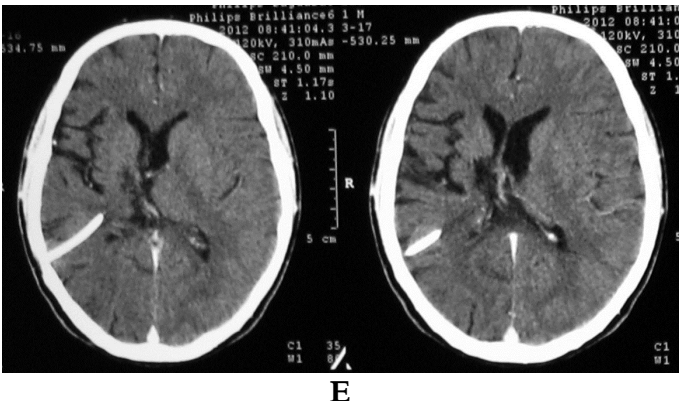

Figure 1 Cerebral CT-scan images on a 22 years old male patient with thalamic abscess; A - native preoperative image; $\mathrm{B}$ - preoperative image with contrast agents; $\mathrm{C}$ - postoperative image; D postoperative image at 3 weeks postoperative showing porencephalic cyst; E - postoperative image after cyst drainage.

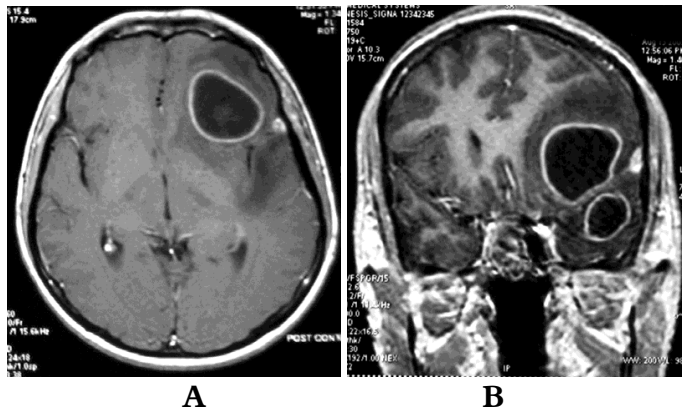



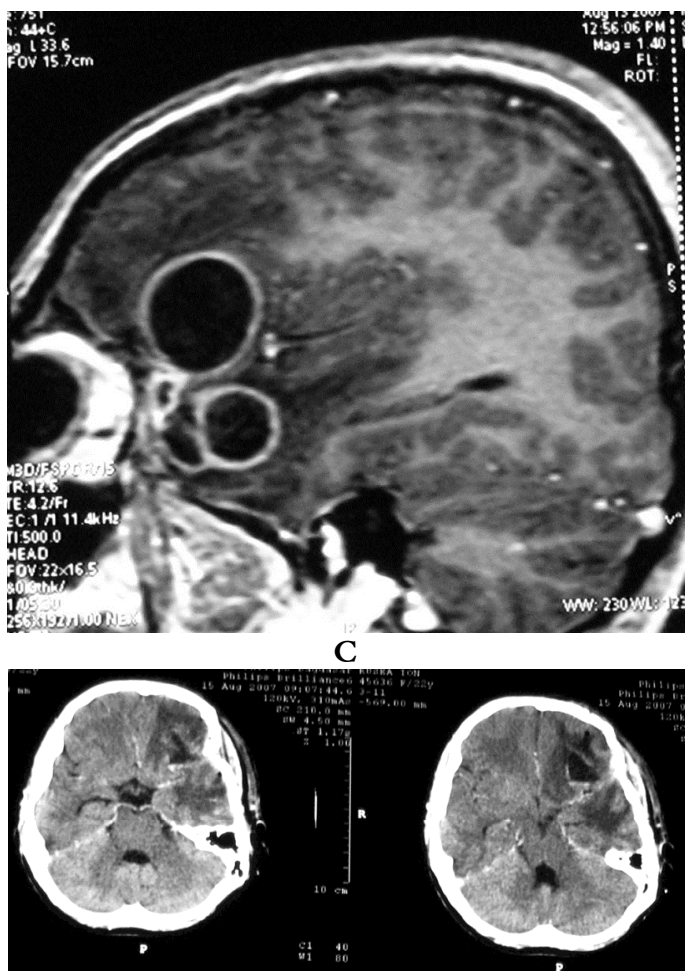

D

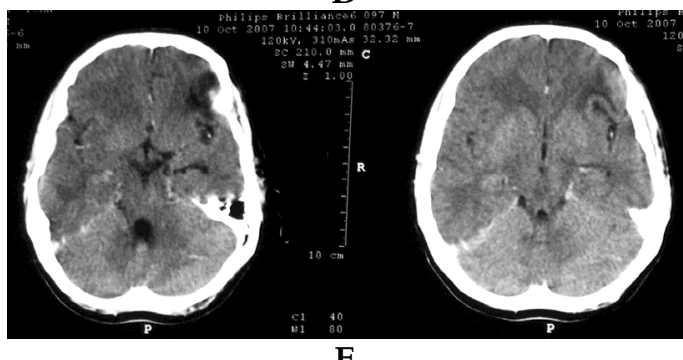

E

Figure 2 Cerebral IRM images on a 24 years old male patient with 2 abscesses - one frontal, one temporal - on the left side; $\mathrm{A}$ - preoperative image, axial view; $\mathrm{B}$ - preoperative image, coronal view; C preoperative image, sagittal view; $\mathrm{D}$ - postoperative

image (CT scan); E - postoperative image at 2 months (CT scan).

\section{Discussion}

Management of cerebral abscess consists actually by the next methods: medical treatment and surgical treatment (18). Antibiotherapy is a clue factor in the healing of the patient. The choice of antibiotics for any bacterial abscesses depends upon a number of factors, the most important being the causative organism. For this reason, we recommend to take samples directly from the abscess itself before initiating antibiotic therapy.

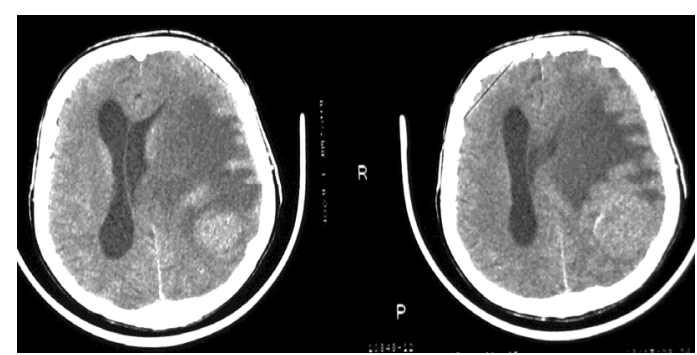

A

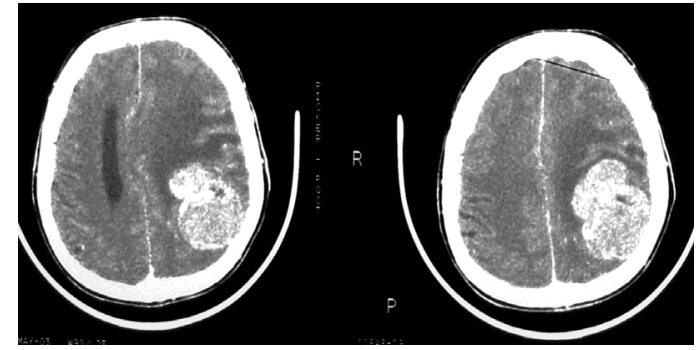

B

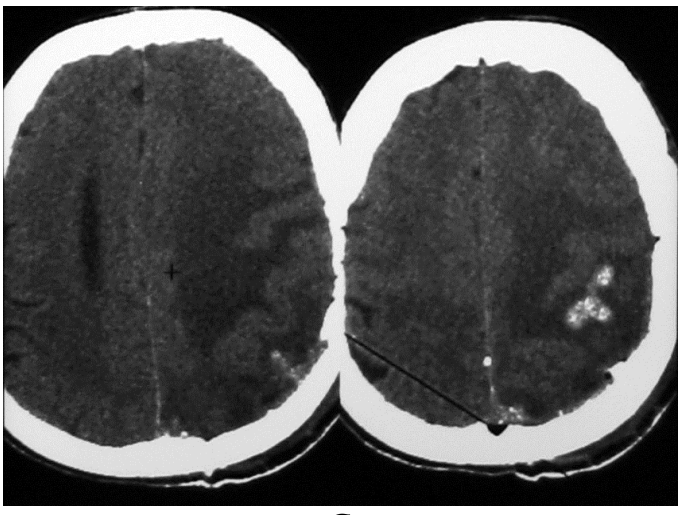

C

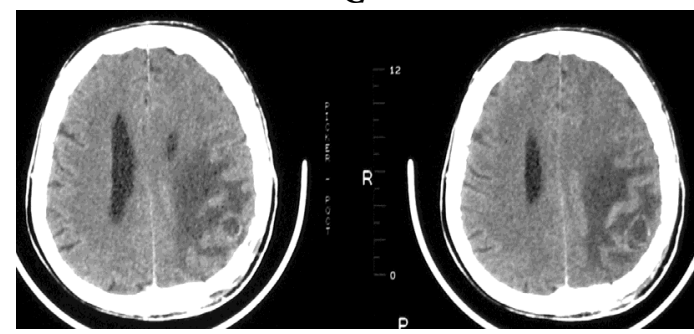

D 


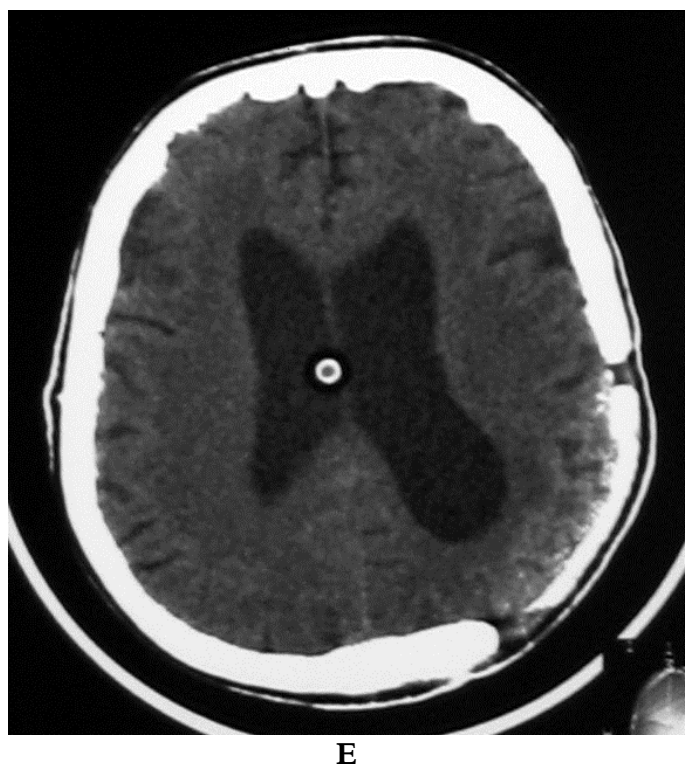

Figure 3 Cerebral CT-scan images on a 57 years old female patient with 2 parietal abscesses after surgery for a brain tumor. A - native preoperative image; $\mathrm{B}$ preoperative image with contrast agents showing a brain tumor; $\mathrm{C}$ - postoperative image; D postoperative image at 1 month showing brain abscesses; E - postoperative image.

Other factors that relate to antibiotic selection are the penetration into the abscess cavity, the bactericidal or bacteriostatic capacity of the antibiotic, and its bacteriologic spectrum. In relation to these properties, many studies have demonstrated that antibiotics that cannot penetrate the blood-brain barrier under normal conditions, but they can do so in an area of inflammation. No abscess greater than $2.5 \mathrm{~cm}$ in diameter responded to antibiotic therapy. These findings led to the recommendation of surgical drainage of any abscess with a diameter greater than $3 \mathrm{~cm}$ (5).

Currently, the operative management of cerebral abscesses is based upon two separate procedures: burr hole aspiration, stereotactic biopsy, stereotactic drainage, and complete excision. Aspiration has been shown to produce excellent results in a number of series. It allows for precise localization and decompression of abscess cavities with minimal tissue damage. This is an obvious benefit when the neurosurgeon is dealing with deeply seated lesions, with those located in eloquent areas, or with multiple abscesses. Often aspiration can be performed with the patient under local anesthesia, which reduces the surgical risk. Excision also has advantages in specific circumstances and has been proven successful in various series $(10,20)$. Cerebellar abscesses should be explored by craniectomy, because any failure of treatment in this region can be rapidly fatal (1). The two most feared complications of cerebral abscess are herniation secondary to mass effect and abscess rupture into the ventricle and subarachnoid space (14). We did not record any rupture into the ventricles, but four cases with important edema, required decompressive craniotomy and corticotherapy.

Late recurrence abscesses could be considered complication, as it is usually due to either inadequate medical or surgical treatment, following complete resections. Recurrence is more common after aspiration alone (3-25\%) than after excision $(0-6 \%)(15)$. This is the main reason for our policy of surgical treatment.

The most common mechanism of infection was hematogenous spread $(35.71 \%, \mathrm{n}=30)$, a factor which in our series relates to the poorest prognosis. The route of transmission was contiguous infection in $33.33 \% \quad(n=28)$ cases and determined which region of the brain was affected. Sinusitis was associated with frontal lesions, whereas otogenic infections were associated with temporal or cerebellar 
DOI: $10.2478 / \mathrm{v} 10282-012-0008-\mathrm{z}$

abscesses. Abscesses with unknown underlying causes accounted for $23.8 \%$ $(n=20)$ of patients in our series, which is a rate comparable to the lowest values (15$48 \%)$ reported in the literature $(7,17,19)$.

The prognosis for patients with intracerebral abscesses is highly related with their neurological condition and size of the abscess at the time of admission in large series (3, 11, 13). Neurological sequels from brain abscess declined from 16 to $52 \%$ in most series before 1975 to about 4 to $27 \%$ in series since then (6). These sequels fall into three major categories: focal neurological deficits, cognitive impairment and seizures. In our series six cases remained with permanent motor deficit $(7.14 \%)$, and four $(24.76 \%)$ with controllable seizures. In our study, hematogenous spread and advance age were predictors of poor outcome and our findings were similar to results from recent works (8). In our series, $82.14 \%$ of patients had a favorable outcome without sequels, compared with the outcomes reported by other authors $(17,19)$.

The relevant studies from the literature report mortality rate ranging from 0 to $21 \%$ in alert patients, to $60 \%$ in patients with signs of cerebral herniation, to $89 \%$ for those in comatose state, and $32-42 \%$ in patient with cardiac anomalies and brain abscesses (8). At present, major centers report a mortality rate of less than $10 \%(8$, $20)$. In our series we had six deaths $-7.14 \%$, lower than the values reported for other series $(4,13,17)$. Out of the total deaths, $66.66 \%(n=4)$ were due to medical causes. Out of a total $33.33 \% \quad(n=28)$ of complications, $64.28 \%$ were associated with medical causes not directly related to surgery.

\section{Conclusions}

In recent years, the drop in mortality rate and improved outcome had been due to the usage of CT scanning, improved bacteriologic techniques, more versatile antibiotics, and introduction of stereotacticguided surgery.

Complete excision state that this method allows diminishing the period of time of antibiotic treatment following surgery (from 4-6 weeks to 2-4 weeks). Our findings are similar to the results of recent works, although in our series, there is a higher frequency of aerobe germs.

Despite of a poor medical and biological condition, the patients with brain abscesses, outside of HIV infections, benefit from neurosurgical adequate treatment, and if supportive medical and general therapy is continued and sustained, healing and survival in good neurological status is the rule.

\section{References}

1. Carpenter JL (1994). Brain stem abscess: cure with medical therapy, case report, and revue. Clin Infect Dis. 18 (2): 219-26;

2. Carpenter J, Stapleton S, Holliman R (2007). Retrospective analysis de 49 cases of brain abscess and review of the literature. Eur J Cin Microbiol Infect Dis, 26:1-11;

3. Cavusoglu H, Kaya RA, Turkmenoglu ON, Colak I, Aydin Y (2008) Brain abscess: analysis of results in a series of 51 patients with a combined surgical and medical approach during an 11-year period. Neurosurg Focus 24:E9;

4. Chang YT, Lu CH, Chuang MJ, Huanh CR, Chuang YC, Tsai NW, Chen SF, Chang CC, Chang WN (2010). Supratentorial deep-seated bacterial brain abscess in adults: clinical characteristics and therapeutic outcome. Acta Neurol Taiwan 19:178-183;

5. Gormley W, Rosenblum ML (1996). Cerebral abscess. In; Tindall TG, Cooper PR, Barrow DR, eds. The practice of Neurosurgery, Baltimore; Williams and Wilkins pub, 3343-3354;

6. Korinek AM (1994). Cerebral abscess and empiema, Rev Prat. 44 (16): 2201-5;

7. Kothari M, Goel A (2008). Brain abscess: a cogent clarifier of the confused concept of immunity. Neurosurg Focus 24 (6):E16;

8. Landriel F, Ajler P, Hem S, Berdersky D, 
Goldschmidt E, Garategui L, Vecchi E, Carrizo A (2012). Supratentorial and infratentorial brain abscesses: surgical treatment, complications and outcome - a 10year single-center study, Acta Neurochir. 154:903-911; 9. Mamelak AN, Obana WG, Flaherty JF, Rosenblum ML (1994). Nocardial brain abscess: treatment strategies and factors influencing outcome. Clical study. Neurosurgery 35(4):622-631;

10.Mamelak AN, Mampalam TJ, Obana WG, Rosenblum ML (1995). Improved management of multiple brain abscesses: a combined surgical and medical approach. Neurosurgery, 36 (1): 76-85;

11.Martinez Vazquez C (1994). Cerebral abscess. The clinical and evolutionary aspects of 17 cases treated surgically, An Med Interna, 11 (6): 268-72;

12.Mathisen GE, Jonson JP (1997). Brain abscess. Clin Infect Dis. 25:763;

13.Nathoo N, Nadvi SS, Narotam PK, van Dellen JR (2011) Brain abscess: management and outcome analysis of a computed tomography era experience with 973 patients. World Neurosurg 75(5-6):716-726;

14.Obana WG, Rosenblum ML (1993). Surgery for intracranial infections. In: Post KD, Friedman E,

McCormick P, eds. Postoperative complications of neurological surgery. New York: Thieme Medical publishers, 146-156;

15.Rajshekhar V, Chandy MJ (1994). Successful stereotactic management of a large cardiogenic brain stem abscess. Neurosurgery. 34 (2): 368-371;

16.Tattevin P, Bruneel F, Clair B, Lellouche F, de Broucker T, Chevret S, Bedos JP, Wolff M, Regnier B (2003). Bacterial brain abscesses: a retrospective study of 94 patients admitted to an intensive care unit (1980 to 1999). Am J Med. 115:143;

17.Tseng JH, Tseng MY (2006). Brain abscess in 142 patients: factors influencing outcome and mortality. Surg Neurol 65:557-562;

18.Wispelwey B, Scheld WM (1991). Brain abscess. In: Scheld W, Whitley R, Durack D, eds. Infections of the central nervous system. New York: Raven Press, 457486;

19.Xiao F, Tseng MY, Teng LJ, Tseng HM, Tsai JC (2005) Brain abscess: clinical experience and analysis of prognostic factors. Surg Neurol 63(5):442-449;

20.Yang SY (1981). Brain abscess; a review of 400 cases. J Neurosurg. 55:794-799. 